\title{
Querying Registry-Published Mathematical Web Services
}

\author{
Rebhi Baraka* Wolfgang Schreiner \\ Research Institute for Symbolic Computation (RISC) \\ Johannes Kepler University, Linz, Austria \\ \{rbaraka,schreine\}@risc.uni-linz.ac.at
}

\begin{abstract}
This paper describes a light-weight, content-based, functional query language. The Mathematical Services Query Language (MSQL) has been developed for querying mathematical web services described in the Mathematical Services Description Language (MSDL) and published in the MathBroker registry. Based on a client query, MSQL uses the registry to retrieve a candidate collection of documents and then uses its own querying functionality to filter these documents based on their contents.
\end{abstract}

\section{Introduction}

Describing, publishing, and discovering web services are crucial issues that have recently received considerable attention. A mathematical service is a web service that offers the solution to a mathematical problem (based on e.g. a computer algebra system or on an automated theorem prover). In our MathBroker project [9], the XML-based Mathematical Services Description Language (MSDL) [4] has been developed to adequately describe mathematical services respectively their constituent entities such as problems, algorithms, implementations, and machines. To facilitate the process of publishing and discovering mathematical services, we have developed the MathBroker registry [3] where MSDL descriptions of services are published such that clients can discover them by browsing or querying it.

Figure 1 illustrates the MathBroker information model for the description of mathematical web services. It shows the kinds of entities that constitute the description of a service and the associations among them. The entities are:

- Problem that can be specified by input/output parameters and input/output conditions. A problem can be a special version of another problem.

*This work was sponsored by the FWF Project P17643-NO4 "MathBroker II: Brokering Distributed Mathematical Services".
- Algorithm that can be described by (a link to the description of) the problem it solves, as well as by time and memory complexity, and termination conditions.

- Implementation that can be described by the software used for implementing an algorithm and for the resulting runtime efficiency (absolute efficiency factors for the algorithmic complexity).

- Realization that brings together the abstract specification of the service functionality with the actual details of the interface described in the Web Services Description Language (WSDL).

- Machine that can be described by its processor type and speed, by its memory size, and by the type of the operating system it uses.

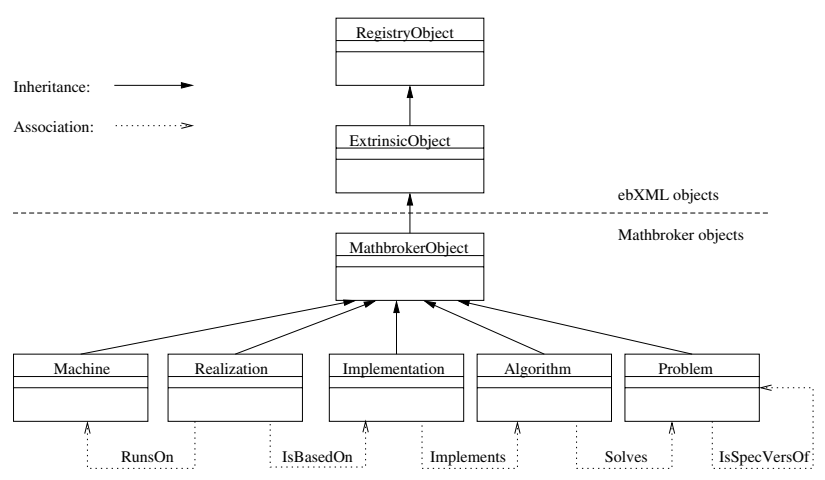

Figure 1. The MathBroker Information Model

A skeleton of a service description in MSDL containing these entities and their associations is shown in Figure 2. The associations are fixed within the description (closed world assumption) because algorithms are designed to solve specific problems, implementations realize specific algorithms, etc. so that it is not the case that additional associations are established by external sources. Mathematical content in this description is written in OpenMath [11] 


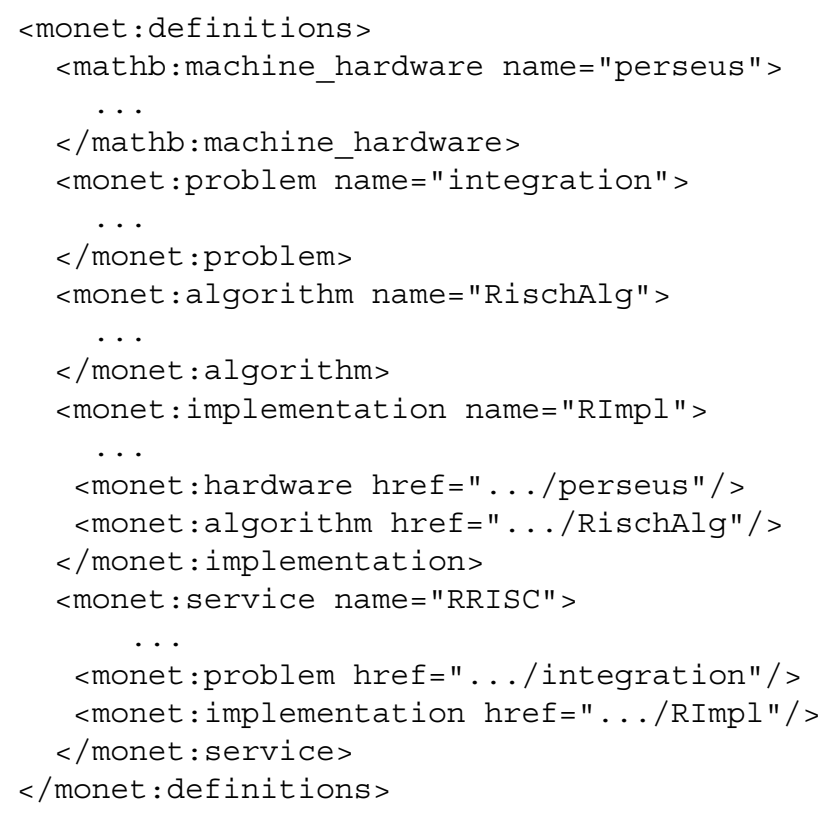

Figure 2. A Skeleton of a Service Description

which is an XML-based format for representing mathematical objects in a semantics-preserving way.

The MathBroker registry implementation incorporating the information model provides a set of functionalities for processing, classifying, associating, publishing, and searching MSDL service descriptions in the registry. Service descriptions are classified according to a predefined classification taxonomy which represents a tree-structured way to categorize descriptions. A common example of a classification in mathematics is the GAMS (Guide to Available Mathematical Software) classification taxonomy.

Searching facilities of the MathBroker registry are rather limited, they only allow to query metadata accompanying the service when published in the registry. Although metadata such as the name, the unique identifier, the classifications, and the associations of a service are useful in some cases, e.g. when we are seeking a service based on its associations to other entities, they are insufficient in many cases involving mathematical services as a basis for service discovery, e.g., when we are seeking a service whose problem has a certain input precondition. Therefore it is necessary to resort to the contents of the MSDL description of a service where the complete information can be found. To overcome this limitation of the current search facilities of the registry, we have designed and implemented the Mathematical Services Query Language (MSQL) for querying the contents of the MSDL documents that are published in a registry.

MSQL has a set of features compatible with today's XML query languages to make the process of querying MSDL documents easy and efficient. The resulting simple, light-weight, and content-based query language is currently being extended to deal with the semantic content of MSDL by automated reasoning techniques.

The rest of this paper describes the architecture (Section 2), the features (Section 3), and the implementation (Section 4) of MSQL.

\section{The MSQL Architecture}

Figure 3 provides a high-level overview of the MSQL architecture which consists of the following parts:

- The MSQL Engine which constitutes the querying functionality.

- The MathBroker Registry where MSDL documents are published.

- The Reasoner which handles the part of a query that needs reasoning (currently being implemented).

A client application sends a query to the engine and receives a set of MSDL descriptions. These descriptions are either returned to the user or processed further for specific tasks, e.g., to access the service having the resulting description.

The MSQL Engine consists of the following components which are designed according to the query structure explained in Section 3:

- The Query Processor receives the query, divides it into processable parts, and hands each part to its corresponding component.

- The Registry Handler receives from the processor the query part needed to retrieve documents from the registry based on their types and classifications.

- The Expression Evaluator evaluates the expression part of a query against the documents retrieved from the registry, filters them, and forwards those passing the filter to the Result Quantifier and Sorter.

- The Reasoner Interface receives from the processor the query part that needs semantic reasoning and sends it to the reasoner.

- The Result Quantifier and Sorter decides whether to return some or every queried document as a result and whether to sort the resulting documents.

In this architecture, an MSQL query received from a client is processed as follows:

- The query is parsed according to MSQL grammar and transformed into an abstract syntax tree.

- The engine connects to the registry and invokes the "MathBroker Query Manager" which is part of the registry API. It queries the registry for candidate documents based on the type of document required and 


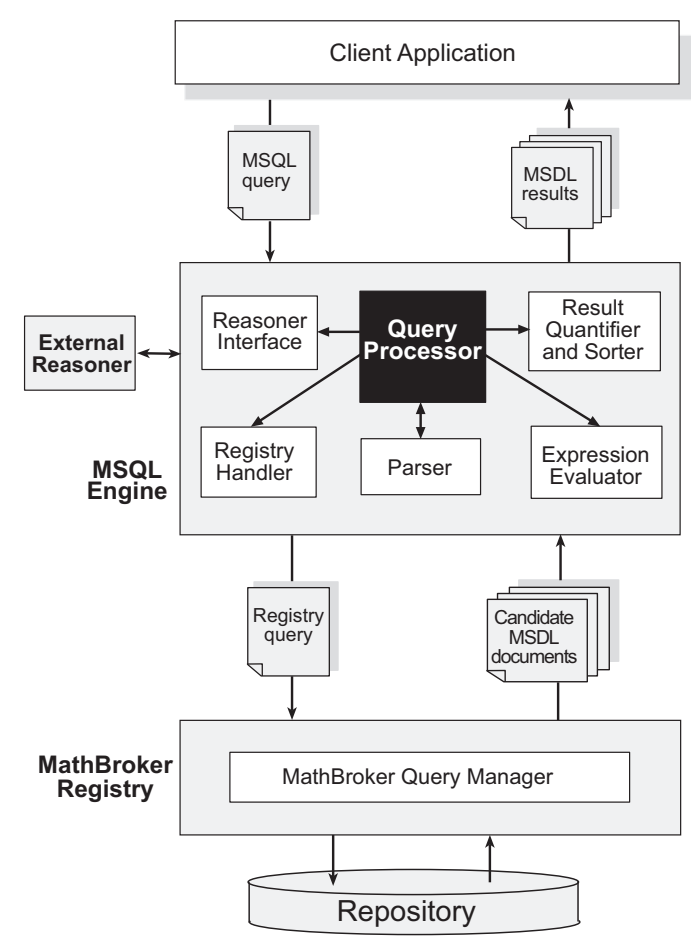

Figure 3. The MSQL Architecture

based on the classification node under which the required document is classified. It returns to the engine the collection of candidate MSDL documents.

- The Expression Evaluator then evaluates the condition expression of the query against each candidate document and if the document satisfies the condition adds it to the collection of resulting documents.

- The Result Quantifier and Sorter then quantifies and orders the resulting documents depending on the kind of query (see the next section).

The engine then returns the resulting MSDL documents to the client application.

\section{The MSQL Language}

\subsection{Motivation}

Given a set of MSDL documents (of a structure such as the one in Figure 2) published in the registry, we would like to perform queries of the following kind:

Find every problem under classification concept "/GAMS/Symbolic Computation" whose first input argument has type "integer".

To accomplish this request, we can do the following:
- consider the registry classification node "/GAMS/Symbolic Computation" and fetch each document with entity type "problem" beneath it.

- process each document and return it, if it satisfies the following criteria: the first "input" occurring in the "problem" has type "integer".

The returned documents may need to be sorted according to some criterion before being returned as a result.

The first step involves contacting the registry and fetching the candidate documents. The second step involves processing the returned candidate documents to see if they satisfy the stated criteria. Based on these steps we have designed the language described in the next subsection.

\subsection{The Query Structure}

The general structure of a query in MSQL is:

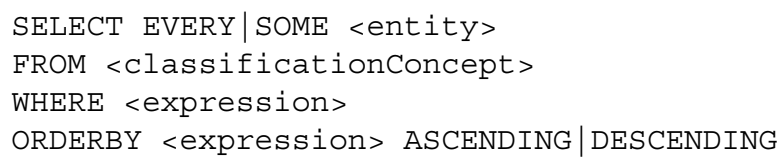

The query has four main clauses: the SELECT clause, the FROM clause, the WHERE clause, and the ORDERBY clause. The FROM clause and part of the SELECT clause, namely entity, are registry-oriented, i.e., their functionality is applied to the registry. They determine the type and the classification of document and retrieve it from the registry. This limits the range of documents to be queried to those who are of type entity and classified under classificationConcept. The entity types as stated in the information model (see Figure 1) are PROBLEM, ALGORITHM, IMPLEMENTATION, REALIZATION, and MACHINE. ClassificationConcept is a node in a given classification taxonomy of the registry, e.g., "/GAMS/Symbolic Computation" in the GAMS classification of mathematical subjects. The SELECT clause also determines whether to return some or all of the resulting documents to the user by its SOME or EVERY clause.

The WHERE and the ORDERBY clauses apply their expression parts to each candidate document retrieved from the registry. The expression of the WHERE clause is a logical condition: if it is evaluated to true, the document is considered as (part of) the result of the query. The ORDERBY clause sorts the resulting documents in ASCENDING/DESCENDING order based on the stated criteria. We illustrate the structure described so far by an example.

Example 1. Find all problems under the classification concept "/GAMS/Symbolic Computation" with first input of type integer and order them according to their names in descending order.

SELECT EVERY problem 
FROM /GAMS/Symbolic Computation

WHERE //body/input [1]/signature/om:OMOBJ/

om:OMS [1] [ ( ( @name = "Z" )

and ( @cd = "setname1" ))]

ORDERBY /problem/@name descending

This query asks for every "problem", i.e., every document of type "problem" classified under "/GAMS/Symbolic Computation" that satisfies the WHERE expression. The resulting documents are to be sorted in descending order according to their names. The core of the query is its WHERE expression which allows us to express the first input and check its type (details is given below).

Since we are designing a light-weight query language, we have specified a minimal set of expressions that are necessary to address the contents of the target MSDL documents. MSQL expressions include: path expressions that can access every part of an MSDL document; expressions involving logical, arithmetic, and comparative operators; conditional expressions; quantified expressions; functions; and variable bindings. The formula is applied to an MSDL document modeled as a tree of nodes. A node can be a root, an element node (element), an attribute, or a text. The various kinds of expressions are described below.

\section{Path Expressions}

These expressions are special XPath [6] expressions. A path expression is used to locate nodes within a document tree. It consists of a series of one or more steps, separated by "/" or "//", and optionally starting with "/" or " //".

A " $/$ " at the start of a path expression begins the path at the root of the current node.

A " $/ /$ " at the start of a path expression begins a sequence that contains the root of the current node plus all nodes descending from it. This node sequence is used as the input to subsequent steps in the path expression. The / / body step in Example 1 traverses the root node and nodes descending from it until the body node which is used as input to the /input [1] step.

A step generates a sequence of items and then filters the sequence by zero or more predicates. The value of the step consists of those items that satisfy the predicates.

A predicate consists of an expression enclosed in square brackets. The predicate expression can either be logical which evaluates to a truth value or numeric which evaluates to a numeric value. In Example 1, the predicate in step / input [1] is numeric, it specifies the first input node.

The /om:OMS [1] [(@name = ' ' $\mathrm{Z}^{\prime \prime}$ ) and (@cd = ' 'setname1'')] step has a sequence of two predicates. The first predicate is numeric and it specifies the first om: OMS node. The second is logical that uses the = and the and operators to evaluate to a truth value. It operates on the om: OMS node to check if its name attribute has value equal to $\mathrm{Z}$ and $\mathrm{cd}$ attribute has value equal to setname1. The value of the second predicate represents the value of the expression; if its value is true then the current document is selected.

\section{Operators}

MSQL supports arithmetic, logical, and comparison operators for use within predicates. In Example 1, the expression in the last predicate uses the comparison operator "=" and the logical operator "and" to evaluate to a truth value.

\section{Functions}

In the context of a predicate, functions may be applied to the current node to extract information used in some operations.

Example 2. Find all problems in "/GAMS/Arithmetic, error analysis/Integer" that have no precondition.

SELECT EVERY problem

FROM /GAMS/Symbolic Computation

WHERE //body [empty(/pre-condition)]

In this example, the empty function takes a path expression and returns true if the node pointed to by the path is empty. The query uses this function to check if the "body" node has an empty "pre-condition" element node.

\section{Conditional Expressions and Variable Bindings}

Conditional expressions are used when the document to be returned depends on some condition. An expression or a value that is used in more than one place in a query can be bound to a variable so it does not need to be defined again.

Example 3. Find every service in "/GAMS/Linear Algebra" such that if it has an implementation it runs on a machine called perseus or its interface is on the said machine.

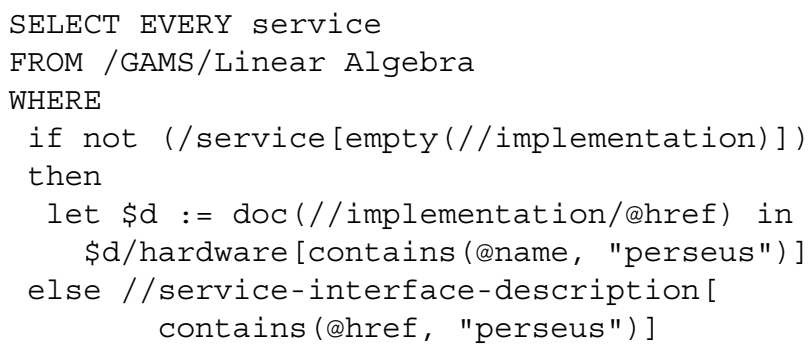

Example 3 shows a query that uses a conditional expression to decide if the current service document node has (IsBasedOn) an implementation. If this is the case, it takes the URI of such implementation document and retrieves it from the registry and checks if this implementation is related to the machine perseus. If this is not the case, it checks (in the else) if the service has its interface on the 
said machine, i.e., Runs on on the said machine. The let clause is used to bind a document to variable $d$. Variable $d$ is then used as part of the path expression.

In the same example, the contains function returns true if its first argument value contains as part of it its second argument value. The doc function returns the root node of the document whose name appears as its argument. Its argument is a URI that is used as the address of the required document in the registry.

The query in Example 3 aside from showing the expressiveness of MSQL in dealing with the MSDL content, it also reveals how MSQL utilizes the structure of the MSDL information model (see Figure 1) supported by the registry. It uses associations (e.g., IsBasedOn, RunsOn) among the entities of the model implicitly in the query.

\section{Quantifiers}

MSQL provides universal and existential quantifiers to test if every/some element in a document satisfies a condition.

Example 4. Find all problems in "/GAMS/Arithmetic, error analysis/Integer" in which the OpenMath content dictionary "sts" and the "mapsto" symbol are used in the same signature.

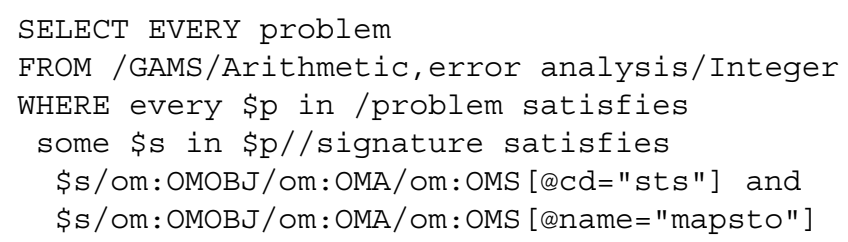

The every quantifier requires all "problem" nodes to satisfy the "some" quantifier which checks if at least one signature satisfies the condition specified.

\section{Semantic Expressions}

The above MSQL expressions are performed on the syntactic structure of documents. We are currently working to extend MSQL to provide also semantic queries.

Example 5. Find a problem whose input $\mathrm{x}$ and output $\mathrm{y}$ satisfy the condition $\operatorname{diff}(y)=x$ ('diff' means symbolic differentiation).

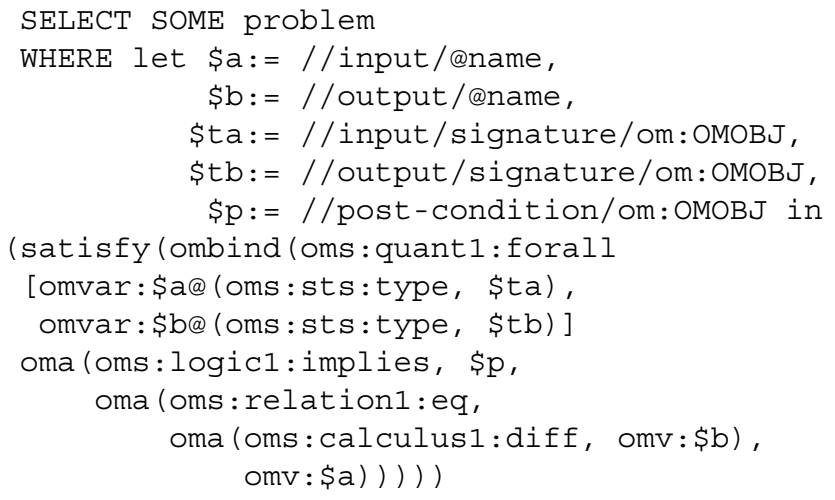

The satisfy expression represents the quantified formula: $\forall \$ a: \$ t a, \$ b: \$ t b(\$ p \Rightarrow \operatorname{diff}(\$ b)=\$ a)$ where meta-variables $\$ a$ and $\$ b$ represent input/output variables of the specification, $\$$ ta and $\$ t b$ represent their types, and $\$ p$ represents the post-condition of the specification. The query engine will substitute the actual content of the specified document for these meta-variables and forward the resulting (OpenMath) formula to the reasoner (See Figure 3) which returns the decision about its truth to the engine.

Thus the MSQL engine combines syntactic queries on MSDL documents with semantic queries on their interpretation in some mathematical domains.

\section{The MSQL Implementation}

The implementation of MSQL is based on a formal semantics [1]. We have used the format of denotational semantics [12] to give formal meaning to each construct defined in the previous section. In the implementation, each mathematical function mapping a syntactic domain to a semantic domain is realized by a Java method whose body corresponds to the mathematical function definition.

In the implementation of the MSQL engine architecture (see Figure 3) the main functionality is exposed to the user by the MSQL API [2]. The following code outlines one way for using the API in client applications:

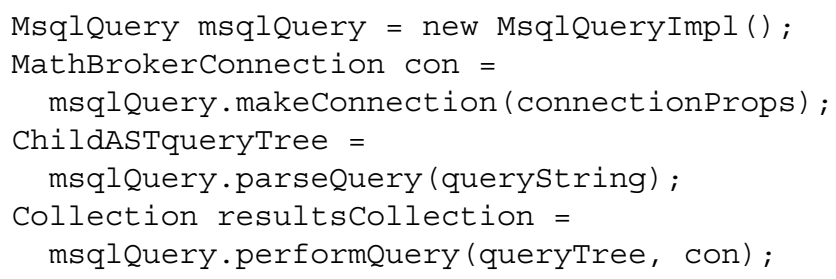

The interface MsqlQuery presents the MSQL engine and contains the functionality for accessing the rest of the API. A connection is made to the registry through the makeConnection method. A received MSQL query is parsed using the parseQuery method. The query is processed and the results are returned all together as a collection using the performQuery method.

An alternative to performQuery is iterateQuery which allows the client to iteratively ask for one document satisfying a query after the other (such that the engine needs not process all candidate documents when the client is satisfied with some result early).

We have tested MSQL engine with different examples representing the various functionalities of MSQL and the expected scenarios of its usability in the MathBroker framework. However, the overall evaluation of the engine, e.g., in terms of scalability needs further investigation. 


\section{Related Work}

Existing XML query languages range from ones that support simple node finding and path expressions to more comprehensive ones that support processing, transformation, and querying tasks of XML documents. QUILT [5] is a language that attempts to unify concepts from some of these query languages in order to exploit the full versatility of XML. Its proposal has been adopted latter as the basis for the development of XQuery [7]. XQuery is intended primarily as a query language for querying collections of XML documents or documents viewed as XML (e.g., a SOAP representation of any data source). The language is designed to be broadly capable of dealing with many sources of XML and not only query them, but also process them, and have new XML structures as a result.

Unlike XQuery, MSQL is not intended to be a general purpose XML query language. It is a domain-specific querying language that addresses the querying and discovery requirements of the MathBroker framework. Furthermore, although XQuery has a rich functionality, it cannot address some of our requirements such as dealing with classification schemes and types of objects stored in the MathBroker registry. So, instead of using an XQuery based query engine that might not be compatible with our semantic extensions and might induce extra overhead, we designed and implemented our own query language maintaining a uniform framework. However, we used some features of XQuery and of its predecessors, e.g., MSQL adapts from XQuery the syntax for navigating in the hierarchical structure of MSDL documents. From SQL (Structured Query Language), MSQL utilizes the idea of a series of clauses based on keywords that provide a query pattern (the SELECT-FROM-WHERE pattern in SQL).

At the semantic level of information representation and searchability for XML, there exists a number of languages such as RDF and OWL and a number of query tools for each kind of representation. The use of these tools in describing and discovering mathematical content is currently being investigated in another part of our MathBroker project [9].

Considering mathematical-oriented tools, there is a number of approaches [8] for searching mathematical content that range from basic textual methods to semantic-driven techniques. Their functionality depends on the representation of the target documents. One approach [10] for achieving service discovery performs matchmaking between representations of tasks (client requests) and capabilities (service descriptions). The approach applies a normalization process on a task. It then compares the normalized task with a registered capability calculating a similarity value that is used in the matchmaking process. This approach covers certain semantical aspects of service descriptions but is ultimately limited in its power.

\section{Conclusion}

The Mathematical Services Query Language (MSQL) is a light-weight, content-based, functional query language developed for querying mathematical descriptions given in the Mathematical Services Description Language and published in the MathBroker registry. MSQL complements the metadata-based querying facility of the registry.

MSQL supports syntax-based queries on the syntactic structure of mathematical service specifications. However, we are currently working to extend MSQL to semantic based querying where the engine contacts a reasoner that operates on the underlying semantical structure of MSDL.

\section{References}

[1] R. Baraka. Mathematical Services Query Language: Design, Formalization, and Implementation. Technical report, RISC, Austria, September 2005. See ftp://ftp.risc.unilinz.ac.at/pub/techreports/.

[2] R. Baraka. Mathematical Services Query Language (MSQL) API. Research Institute for Symbolic Computation (RISC), September 2005. See http://poseidon.risc.unilinz.ac.at:8080/results/msql/doc/index.html.

[3] R. Baraka, O. Caprotti, and W. Schreiner. A Web Registry for Publishing and Discovering Mathematical Services. In Proceedings of IEEE Conference on e-Technology, eCommerce, and e-Service, Hong Kong, March 29 - April 1, 2005. IEEE Computer Society.

[4] O. Caprotti and W. Schreiner. Towards a Mathematical Service Description Language. In International Congress of Mathematical Software 2002, Bejing, 17-19 August, 2002. World Scientific Publishing.

[5] D. Chamberlin, J. Robie, and D. Florescu. QUILT: An XML Query Language for Heterogeneous Data Sources. In WebDB 2000 Conference. Springer-Verlag, 2000.

[6] W. W. W. Consortium. XML Path Language (XPath) 2.0. W3C Working Draft, April 2005. See http://www.w3.org/TR/xpath20/.

[7] W. W. W. Consortium. XQuery 1.0: An XML Query Language. W3C Working Draft, April 2005. See http://www.w3.org/TR/xquery/.

[8] I. Dahn and A. Asperti. Mathematical Knowledge Management and Searchability. Deliverable D5.4, 2001. See http://monet.nag.co.uk/mkm/MKMNetTN-D5-4.pdf.

[9] MathBroker II: Brokering Distributed Mathematical Services. Research Institute for Symbolic Computation (RISC), September 2005. See http://www/research/parallel/projects/mathbroker2/.

[10] W. Naylor and J. Padget. Semantic Matching for Mathematical Services. In Proceedings of the Forth International conference on Mathematical Knowledge Management, Bremen, Germany, 15 - 17 July, 2005. Springer.

[11] The OpenMath Standard, September 2005. See http://www.openmath.org/cocoon/openmath/index.html.

[12] D. A. Schmidt. Denotational Semantics - A Methodology for Language Development. Allyn and Bacon, 1986. 